\title{
THE GRADED RING OF QUANTUM THETA FUNCTIONS FOR NONCOMMUTATIVE TORUS WITH REAL MULTIPLICATION
}

\author{
MARIYA VLASENKO
}

\begin{abstract}
For quantum torus generated by unitaries $U V=e(\theta) V U$ there exist nontrivial strong Morita autoequivalences in case when $\theta$ is real quadratic irrationality. A.Polishchuk introduced and studied the graded ring of holomorphic sections of powers of the respective bimodule (depending on the choice of a complex structure). We consider a Segre square of this ring whose graded components are spanned by Rieffel scalar products of Polishchuk's holomorphic vectors as in [5] and [8]. These graded components are linear spaces of quantum theta functions in sense of Yu.Manin.
\end{abstract}

\section{INTRODUCTION}

A quantum torus $A_{\theta}$ with an irrational parameter $\theta \in \mathbb{R} \backslash \mathbb{Q}$ is a transformation group $C^{*}$-algebra $C^{*}(\theta \mathbb{Z}, \mathbb{R} / \mathbb{Z})$ for the group action of $\theta \mathbb{Z}$ on $\mathbb{R} / \mathbb{Z}$ or, equivalently, a universal $C^{*}$-algebra generated by two unitaries $U, V \in A_{\theta}$ satisfying relation $U V=e(\theta) V U$. Here $e(x)=\exp (2 \pi i x)$.

Definition 1. $A_{\theta}$ is a quantum torus with real multiplication if $\theta$ is a real quadratic irrationality, i.e. a real irrational root of a quadratic equation with rational coefficients.

Let $k$ be a real quadratic field. In [1] it is proposed to use quantum tori with real multiplication $A_{\theta}, \theta \in k \backslash \mathbb{Q}$ as geometric objects associated to $k$. This should be compared to consideration of elliptic curves with complex multiplication $E_{\tau}=\mathbb{C}$ / $\Gamma, \Gamma=\mathbb{Z}+\tau \mathbb{Z}, \tau \in k^{\prime} \backslash \mathbb{Q}$ for complex quadratic field $k^{\prime}$. Any endomorphism $\alpha: E_{\tau} \rightarrow E_{\tau}$ is a linear map on the universal covering $\mathbb{C}$, so $\operatorname{End}\left(E_{\tau}\right)$ is identified with the ring of multipliers of the lattice $\Gamma$, that is $\{\alpha \in \mathbb{C} \mid \alpha \Gamma \subset \Gamma\}$. We say that $E_{\tau}$ is an elliptic curve with complex multiplication if $\operatorname{End}\left(E_{\tau}\right)$ is larger then $\mathbb{Z}$, which happens precisely when $\tau$ is a complex quadratic number.

Real multiplication of quantum tori has similar interpretation when we consider morphisms in sense of noncommutative geometry: every element of $\operatorname{End}\left(A_{\theta}\right)$ is by definition an (isomorphism class of) $A_{\theta}-A_{\theta}$-bimodule, finitely generated and projective as left and right module at the same time. Every such isomorphism class $[M] \in \operatorname{End}\left(A_{\theta}\right)$ defines an endomorphism $\phi_{[M]}$ of $K_{0}$-group of $A_{\theta}$ via $[P] \mapsto\left[P \underset{A_{\theta}}{\otimes} M\right]$ for finitely generated projective right $A_{\theta}$-modules $P$. It is shown in [1] that when $K_{0}\left(A_{\theta}\right)$ is identified with the lattice $\Gamma=\mathbb{Z}+\theta \mathbb{Z}$ via trace map, then $\phi_{[M]}$ becomes a multiplication by real number. Moreover, this map

$$
K_{0}: \operatorname{End}\left(A_{\theta}\right) \rightarrow\{\alpha \in \mathbb{R} \mid \alpha \Gamma \subset \Gamma\}, K_{0}([M])=\phi_{[M]}
$$

is surjective. So, $A_{\theta}$ is a quantum tori with real multiplication if and only if $K_{0}\left(\operatorname{End}\left(A_{\theta}\right)\right)$ is larger then $\mathbb{Z}$.

In this paper we construct the graded ring of quantum theta functions $R=\oplus R_{n}$ for quantum torus with real multiplication $A_{\theta}$. The construction is described in Section 6 , were we also prove that $\theta \in k \backslash \mathbb{Q}$ can be chosen such that the ring $R$ is

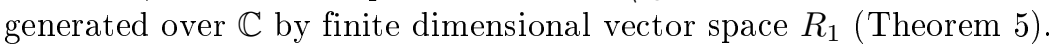


We sketch the definition of $R$ below. First, we need simple facts from number theory. One can prove that $\{\alpha \in \mathbb{R} \mid \alpha \Gamma \subset \Gamma\}=\mathbb{Z}+f O_{k}$ for some integer $f \geq 1$, where $O_{k}$ is the ring of integers of real quadratic field $k=\mathbb{Q}(\theta)$. Thus there are units of infinite order of $O_{k}$ in $\mathbb{Z}+f O_{k}$, and we take one of them $\varepsilon \in\left(\mathbb{Z}+f O_{k}\right) \cap O_{k}^{\times}$. Then there exists a bimodule $M_{\varepsilon}$ with $K_{0}\left(\left[M_{\varepsilon}\right]\right)=\varepsilon$, which is an $A_{\theta}-A_{\theta}$-imprimitivity bimodule. Bimodules of such a kind were studied in [2, 3, [1, and we describe them in Section 5. Sections 1-3 contain discussion of relationship between biprojective bimodules and imprimitivity bimodules. $M_{\varepsilon}$ is an infinite dimensional $\mathbb{C}$-vector space, but one can take finite dimensional subspaces $E_{n} \subset M_{\varepsilon}^{\otimes n}$ of so-called "holomorphic" vectors, and they are compatible with tensor product: $E_{n} \otimes E_{m} \subset E_{n+m}$ $([2],[3])$. So, we obtain the graded $\operatorname{ring} E=\oplus_{n} E_{n}$ with multiplication defined via tensor product. This ring was studied in 4. The choice of "holomorphic" vectors depend on a complex parameter $\tau \in \mathbb{C}$, formally defining "holomorphic" structure on $A_{\theta}$. In this paper we use structure of imprimitivity bimodules on $M_{\varepsilon}^{\otimes n}$ to obtain quantum theta functions from "holomorphic" vectors (see Section 4 for definition of the structure of imprimitivity bimodule on tensor product). It was already noticed in [5] that operator-valued theta functions appear from imprimitivity bimodules over quantum tori.

We use the definition of quantum theta functions given in [6] and [7]. Let us briefly recall it. Consider Heisenberg group $G_{\theta}$

$$
1 \rightarrow \mathbb{C}^{\times} \rightarrow G_{\theta} \rightarrow \mathbb{C}^{2} \times \mathbb{Z}^{2} \rightarrow 0
$$

acting on elements of quantum torus $A_{\theta}$ by

$$
(\alpha ; \vec{x} ; \vec{m}) \sum_{\vec{n} \in \mathbb{Z}^{2}} a_{\vec{n}} U^{n_{1}} V^{n_{2}}=\alpha \sum_{\vec{n} \in \mathbb{Z}^{2}} e\left(n_{1} x_{1}+n_{2} x_{2}\right) a_{\vec{n}} U^{m_{1}} V^{m_{2}} U^{n_{1}} V^{n_{2}} .
$$

A multiplier $\mathcal{L}$ is any free subgroup of rank 2 in $G_{\theta}$, which is a lift of a free subgroup of rank 2 in $\mathbb{C}^{2} \times \mathbb{Z}^{2}$. We denote by $\Gamma(\mathcal{L}) \subset A_{\theta}$ the vector space of elements fixed by $\mathcal{L}$. All elements of $\Gamma(\mathcal{L})$ are called quantum theta functions with multiplier $\mathcal{L}$. For example, take a lattice $L=\mathbb{Z} \vec{s}+\mathbb{Z} \vec{r} \subset \mathbb{Z}^{2}$, and a matrix $\Omega \in \mathcal{M}_{2} \mathbb{C}$, symmetric $\Omega=\Omega^{t}$ and with positive imaginary part $\Im \Omega>0$. Then $\left(e\left(\frac{1}{2} \vec{s}^{t} A^{t} \vec{s}\right) ; A \vec{s} ; \vec{s}\right)$ and $\left(e\left(\frac{1}{2} \vec{r}^{t} A^{t} \vec{r}\right) ; A \vec{r} ; \vec{r}\right)$ generate a multiplier, where $A=\frac{\theta}{2}\left(\begin{array}{cc}0 & 1 \\ -1 & 0\end{array}\right)+\Omega$. Let us denote this multiplier by $\mathcal{L}=\mathcal{L}(L, \Omega)$. Then $\Gamma(\mathcal{L})$ is $\#\left(\mathbb{Z}^{2} / L\right)$-dimensional $\mathbb{C}$-vector space of elements of the form

$$
\Theta[f](\Omega)=\sum_{\vec{m} \in \mathbb{Z}^{2}} f(\vec{m}) e\left(\frac{1}{2} \vec{m}^{t} \Omega \vec{m}\right) e\left(-\frac{\theta}{2} m_{1} m_{2}\right) U^{m_{1}} V^{m_{2}}
$$

for $f: \mathbb{Z}^{2} / L \rightarrow \mathbb{C}$.

We define graded components of our ring of quantum theta functions as $R_{n}=$ $\Gamma\left(\mathcal{L}\left(c_{n} \mathbb{Z}^{2}, \Omega_{n}\right)\right) \subset A_{\theta}$. Now we explain what are $c_{n}$ and $\Omega_{n}$. Recall we have chosen a unit $\varepsilon \in O_{k}$ and a complex parameter $\tau \in \mathbb{C}$. Now we need them to satisfy some technical conditions, especially $\Im \tau>0$ and $\varepsilon=c \theta+d>0, \varepsilon \theta=a \theta+b$ with $\left(\begin{array}{ll}a & b \\ c & d\end{array}\right) \in S L_{2}(\mathbb{Z})$ and $c>0$. Existence of such an $\varepsilon$ one can get for example from Section 6 . Then $c_{n}$ are defined by $\varepsilon^{n}=c_{n} \theta+d_{n}$ with integer $c_{n}, d_{n}$, or, equivalently, by $\left(\begin{array}{ll}a & b \\ c & d\end{array}\right)^{n}=\left(\begin{array}{ll}a_{n} & b_{n} \\ c_{n} & d_{n}\end{array}\right)$, or by $\sum_{n} c_{n} t^{n}=\frac{c}{t^{2}-(a+d) t+1}$. In particular, the last expression shows that $\left\{c_{n}\right\}$ is an increasing sequence of positive integers, since $a+d=\varepsilon+\frac{1}{\varepsilon} \geq 2$. Now $\Omega_{n}=\frac{1}{c_{n} \varepsilon^{n}} \Omega$ where

$$
\Omega=\frac{i}{2 \Im \tau}\left(\begin{array}{cc}
|\tau|^{2} & -\Re \tau \\
-\Re \tau & 1
\end{array}\right)
$$


If we whish to define a product on $R=\oplus R_{n}$, then usual product in $A_{\theta}$ wouldn't do. It was already noticed in [7] that a product of two quantum theta functions in $A_{\theta}$ is not a quantum theta function as a rule. But our quantum theta functions in $R_{n}$ arise from bimodules $M_{\varepsilon}^{n}$ (Proposition 6.2), so we get another product - a bilinear operation $\star: R_{n} \otimes R_{m} \rightarrow R_{n+m}$, naturally coming from tensor product of bimodules.

So, we get the ring $R$ whose elements are formally an elements of quantum tori $A_{\theta}$, but multiplication law is different from the one in $A_{\theta}$. In fact $R$ is isomorphic to a kind of Segre square of $E$ - the subspace in $E \bar{\otimes} E$ generated by elements $a \bar{\otimes} b$ with $a, b \in E_{n}$ for some $n$. Here $\bar{\otimes}$ means that $(\alpha a) \bar{\otimes} b=a \bar{\otimes}(\bar{\alpha} b)$ for $\alpha \in \mathbb{C}$. Both $R$ and $E$ encapsulate the structure of real multiplication and use arithmetical data to be constructed. But the following question still remains unanswered: whether we can use such a rings to obtain arithmetical invariants of real quadratic field $k=\mathbb{Q}(\theta) ?$

Acknowledgement. I am grateful to Yuri Ivanovich Manin who taught me the idea of real multiplication and collaboration with whom led to writing this notes. I also thank Alexander Polishchuk who read the first version of the paper and re-

marked that our ring of quantum theta functions is a Segre square of the ring of "holomorphic" vectors.

\section{Strong Morita equivalence}

Let $A$ be a pre- $C^{*}$-algebra, i.e. $\mathbb{C}$-algebra with involution and norm satisfying $\|x\|^{2}=\left\|x^{*} x\right\|$ and $\|x\|=0$ if and only if $x=0$ for $x \in A$. If $A$ has a unit element $1 \in A$ it is assumed that $\|1\|=1$. An $A$-valued pre-inner product on linear space $M$ is an $A$-valued sesquilinear form $\langle\cdot, \cdot\rangle$ (here it does not matter in which variable it is conjugate linear) such that $\langle x, x\rangle \geq 0$ in completion of $A$ and $\langle x, y\rangle^{*}=\langle y, x\rangle$ for $x, y \in M$. We denote $\operatorname{Im}\langle\cdot, \cdot\rangle \subset A$ the set of finite sums of elements of the form $\langle x, y\rangle$ for $x, y \in M$.

Following definitions were introduced in 9 .

Definition 1.1. A left A-module $M$ is called a left A-rigged space if it is endowed with A-valued pre-inner product ${ }_{A}\langle\cdot, \cdot\rangle: M \times M \mapsto A$, linear in first argument and conjugate linear in second, such that ${ }_{A}\langle a x, y\rangle=a_{A}\langle x, y\rangle$ for $x, y \in M, a \in A$ and the two-sided ideal $\operatorname{Im}_{A}\langle\cdot, \cdot\rangle$ is dense in $A$.

Note that ${ }_{A}\langle a x, y\rangle=a_{A}\langle x, y\rangle$ for an $A$-valued inner product imply also ${ }_{A}\langle x, a y\rangle={ }_{A}\langle x, y\rangle a^{*}$. That is why $\operatorname{Im}_{A}\langle\cdot, \cdot\rangle$ is two-sided ideal as mentioned. Definition of a right rigged space we obtain by simple reflection from the left to the right:

Definition 1.2. A right $A$-module $M$ is called a right $A$-rigged space if it is endowed with pre-inner product $\langle\cdot, \cdot\rangle_{A}: M \times M \mapsto A$, conjugate linear in first argument and linear in second, such that $\langle x, y a\rangle_{A}=\langle x, y\rangle_{A}$ a for $x, y \in M, a \in A$ and the ideal $\operatorname{Im}\langle\cdot, \cdot\rangle_{A}$ is dense in $A$.

Let now $A, B$ be pre- $C^{*}$-algebras.

Definition 1.3. An $A-B$ bimodule $M$ is called an imprimitivity bimodule if

(1) $M$ is left- $A$-right- $B$-rigged space;

(2) ${ }_{A}\langle x, y\rangle z=x\langle y, z\rangle_{B}$;

(3) $\langle a x, a x\rangle_{B} \leq\|a\|_{A}^{2}\langle x, x\rangle_{B}$ and ${ }_{A}\langle x b, x b\rangle \leq\|b\|_{B}^{2}{ }_{A}\langle x, x\rangle$

for $x, y, z \in M$ and $a \in A, b \in B$.

Note that in imprimitivity bimodule we also have relation ${ }_{A}\langle x, y b\rangle={ }_{A}\left\langle x b^{*}, y\right\rangle$ for any $x, y \in M$ and $b \in B$. Indeed, for $b \in \operatorname{Im}\langle\cdot, \cdot\rangle_{B}$ it is a consequence of 
relation (2) in definition above. Let us check necessary continuity. Suppose $\left\|b_{n}\right\| \rightarrow$ 0 . Then $\left\|{ }_{A}\left\langle y b_{n}, y b_{n}\right\rangle\right\| \leq\left\|b_{n}\right\|^{2}\left\|_{A}\langle y, y\rangle\right\| \rightarrow 0$ by (3). Now by Proposition 2.9 in [9] we have $\left\|{ }_{A}\left\langle x, y b_{n}\right\rangle\right\| \leq\left\|{ }_{A}\langle x, x\rangle\right\|^{\frac{1}{2}}\left\|_{A}\left\langle y b_{n}, y b_{n}\right\rangle\right\|^{\frac{1}{2}} \rightarrow 0$, and analogously $\left\|{ }_{A}\left\langle x b_{n}^{*}, y\right\rangle\right\| \rightarrow 0$. Evidently, symmetrical relation $\langle a x, y\rangle_{B}=\left\langle x, a^{*} y\right\rangle_{B}$ for any $x, y \in M$ and $a \in A$ also holds.

Definition 1.4. ([10]) Two pre-C $C^{*}$-algebras $A, B$ are said to be strongly Morita equivalent if there exist an $A-B$-imprimitivity bimodule.

Example 1.5. Let $A$ be a pre-C ${ }^{*}$-algebra with 1 . Consider $M=A^{n}$ - free right $A$ module of rank $n$. Then End ${ }_{A} M=\mathcal{M}_{n} A-$ the ring of $n \times n$ matrices with entries in $A$, which is a unital pre- $C^{*}$-algebra again. Then $M$ is $\mathcal{M}_{n} A-A$-imprimitivity bimodule with inner products

$$
\begin{gathered}
\langle x, y\rangle_{A}=x^{*} y=\sum_{i} x_{i}^{*} y_{i} \\
\mathcal{M}_{n} A\langle x, y\rangle=x y^{*}=\left(x_{i} y_{j}^{*}\right)_{i, j=1}^{n}
\end{gathered}
$$

Example 1.6. Let $G$ be a locally compact group, and let $H$ and $K$ be closed subgroups of $G$. Let $A=C^{*}(K, G / H), B=C^{*}(H, K \backslash G)$ be transformation group $C^{*}$-algebras for left action of $K$ on $G / H$ and right action of $H$ on $K \backslash G$ correspondingly. It is shown in [10] that there is a natural $A-B$-imprimitivity bimodule $M-$ a completion of the space $C_{c}(G)$ of $\mathbb{C}$-valued continuous functions with compact support on $G$ with respect to an appropriate norm, with inner products given on $f, g \in C_{c}(G)$ by:

$$
{ }_{A}\langle f, g\rangle(k, x)=\beta(k) \int_{H} f(\tilde{x} h) g^{*}\left(h^{-1} \tilde{x}^{-1} k\right) d h
$$

where $\tilde{x} \in G$ is any representative of class $x$, i.e. $x=\tilde{x} H$,

$$
\langle f, g\rangle_{B}(h, y)=\gamma(h) \int_{K} f^{*}\left(\tilde{y}^{-} 1 k\right) g\left(k^{-1} \tilde{y} h\right) d k
$$

where $y=K \tilde{y}$. Here $\beta(\cdot)=\left(\frac{\delta_{G}(\cdot)}{\delta_{K}(\cdot)}\right)^{\frac{1}{2}}, \gamma(\cdot)=\left(\frac{\delta_{G}(\cdot)}{\delta_{H}(\cdot)}\right)^{\frac{1}{2}}, \delta_{G}, \delta_{H}, \delta_{K}$ are the modular functions of locally compact groups $G, H, K$ correspondingly, an involution is defined on $C_{c}(G)$ by $g \mapsto g^{*}(z)=\delta_{G}\left(z^{-1}\right) \bar{g}\left(z^{-1}\right)$, and all integrals above are taken w.r.t. left Haar measures.

We will see in Sections 2,3 below that this two examples are quite similar.

Strong Morita equivalence implies Morita equivalence, i.e. equivalence of categories of Hermitian representations $([9])$. It is not obvious from definitions that strong Morita equivalence is indeed an equivalence relation. In [9] an inverse imprimitivity bimodule is constructed, showing that this relation is symmetric. In Section 4 we define a natural structure of imprimitivity bimodule on tensor product of imprimitivity bimodules for unital $C^{*}$-algebras. In particular it makes evident transitivity of strong Morita equivalence for unital $C^{*}$-algebras.

\section{INNER PRODUCTS FOR PROJECTIVE MODULE}

We generalize Example 1.5 in current section. On a projective module pre-inner products which satisfy all algebraic relations from Definition 1.3 were introduced in [1]. We are going to check the condition of density of images for these inner products now.

Let $A$ be $C^{*}$-algebra with 1 , let $p \in \mathcal{M}_{n} A$ be projection, i.e. $p=p^{*}=p^{2}$. Consider a submodule $M=p A^{n}$ of right $A$-module $A^{n}$ consisting of such columns which are invariant under left multiplication by $p$. Then $\operatorname{End}_{A} M=p \mathcal{M}_{n} A p$, where matrices act by multiplication from the left. $p \mathcal{M}_{n} A p$ is a $C^{*}$-algebra with norm 
restricted from $\mathcal{M}_{n} A$, and since $\|p\|=1$ this is a unital $C^{*}$-algebra. We consider two inner products on $M$ which are restrictions of inner products from Example 1.5

$$
\begin{gathered}
\langle x, y\rangle_{A}=x^{*} y=\sum_{i} x_{i}^{*} y_{i} \\
p \mathcal{M}_{n} A p\langle x, y\rangle=x y^{*}=\left(x_{i} y_{j}^{*}\right)_{i, j=1}^{n}
\end{gathered}
$$

Then $\operatorname{Im}{ }_{p \mathcal{M} A p}\langle\cdot, \cdot\rangle=p \mathcal{M}_{n} A p$, and $\operatorname{Im}\langle\cdot, \cdot\rangle_{A}=\sum_{i, j} A p_{i, j} A$ - the ideal in $A$ generated by matrix entries of $p$.

Proposition 2.1. $p A^{n}$ with inner products defined above is $p \mathcal{M}_{n} A p-A$-imprimitivity bimodule if and only if $\operatorname{Im}\langle\cdot, \cdot\rangle_{A}=A$.

Proof. In unital $C^{*}$-algebra $A$ there is no dense ideal except $A$. So, condition $\operatorname{Im}\langle\cdot, \cdot\rangle_{A}=A$ is necessary for $p A^{n}$ to be right $A$-rigged space. We show it is sufficient. Indeed, all necessary identities for $\langle\cdot, \cdot\rangle_{A}$ and ${ }_{p \mathcal{M} n A p}\langle\cdot, \cdot\rangle$ are satisfied as they are satisfied in Example 1.5] and we already mentioned that $\operatorname{Im}_{p \mathcal{M} n A p}\langle\cdot, \cdot\rangle=$ $p \mathcal{M}_{n} A p$.

In the next section we show that any imprimitivity bimodule between unital $C^{*}$-algebras is of this form. This idea also comes from works of M. Rieffel - one may compare Theorem 10 below to Proposition 2.1 in [1].

\section{IMPRIMITIVITY BIMODULE FOR $C^{*}$-ALGEBRAS With 1}

Theorem 1. Let $A, B$ be two strongly Morita equivalent $C^{*}$-algebras with 1 , and $M$ be a $B-A$-imprimitivity bimodule. Then

(1) $B=\operatorname{End}_{A} M$;

(2) there exist $n \in \mathbb{Z}$, projection $p \in \mathcal{M}_{n} A$ and isomorphism of right $A$-modules $\Psi: M \rightarrow p A^{\infty}$ such that for $u, v \in M:$

$$
\begin{gathered}
\langle u, v\rangle_{A}=\Psi(u)^{*} \Psi(v) \\
{ }_{B}\langle u, v\rangle=\Psi^{-1} \circ \Psi(u) \Psi(v)^{*} \circ \Psi
\end{gathered}
$$

Proof. As $B$ is unital $C^{*}$-algebra, any dense ideal in it is $B$. Then there exist integer $n$ and $x_{1}, \ldots, x_{n}, y_{1}, \ldots, y_{n} \in M$ such that

$$
1_{B}=\sum_{i}{ }_{B}\left\langle x_{i}, y_{i}\right\rangle \text {. }
$$

Consider unital $C^{*}$-algebra $C=\mathcal{M}_{n} A$ and $B-C$-bimodule $N=M^{n}$ consisting of columns of elements of $M$. We define inner products on $N$ by

$$
\begin{aligned}
{ }_{B}\langle m, n\rangle & =\sum_{i}{ }_{B}\left\langle m_{i}, n_{i}\right\rangle \\
\langle m, n\rangle_{C} & =\left(\left\langle m_{i}, n_{j}\right\rangle_{A}\right)_{i, j=1}^{n}
\end{aligned}
$$

One can check $N$ is $B-C$-imprimitivity bimodule. Now ${ }_{B}\langle x, y\rangle=1_{B}$. Consider $z=x\langle y, y\rangle_{C}^{1 / 2}$. Then

$$
{ }_{B}\langle z, z\rangle={ }_{B}\left\langle x, x\langle y, y\rangle_{C}\right\rangle={ }_{B}\left\langle x,{ }_{B}\langle x, y\rangle y\right\rangle={ }_{B}\langle y, x\rangle_{B}\langle x, y\rangle=1_{B},
$$

and $p=\langle z, z\rangle_{C}$ is a projection. Indeed, obviously $p^{*}=p$ and

$$
\langle z, z\rangle_{C}\langle z, z\rangle_{C}=\left\langle z, z\langle z, z\rangle_{C}\right\rangle_{C}=\left\langle z,{ }_{B}\langle z, z\rangle z\right\rangle_{C}=\langle z, z\rangle_{C} .
$$

Consider homomorphism of right $A$-modules $\Psi: M \rightarrow p A^{n}, \Psi(m)=\left(\left\langle z_{i}, m\right\rangle_{A}\right)$, and unital homomorphism of rings $\Phi: B \rightarrow p \mathcal{M}_{n} A p, \Phi(b)=\langle z, b z\rangle_{C}$. We now prove they are both correctly defined and are in fact isomorphisms.

For $\Psi$ consider $j: M \rightarrow N$ given by $j(m)=\left(m \delta_{1 i}\right)_{i=1}^{n}$. Then columns of $\langle z, j(m)\rangle_{C}$ are $\Psi(m), 0, \ldots, 0$. Since $P\langle z, j(m)\rangle_{C}=\left\langle z\langle z, z\rangle_{C}, j(m)\right\rangle_{C}$ $=\left\langle\langle z, z\rangle_{C} z, j(m)\right\rangle_{C}=\langle z, j(m)\rangle_{C}, \Psi(m) \in p A^{n}$. Injectivity of $\Psi$ follows from 
$z\langle z, n\rangle_{C}={ }_{B}\langle z, z\rangle n=n \neq 0$ for nonzero $n \in N$. Surjectivity follows from fact that $\operatorname{Im}\langle z, \cdot\rangle_{C}=p C$.

$\Phi(b)$ is obviously invariant under left ant right multiplication by $p$. To prove injectivity we note that $b={ }_{B}\langle y, x\rangle b_{B}\langle x, y\rangle={ }_{B}\langle b z, z\rangle$, so $b z \neq 0$ if $b \neq 0$. Thus also $\langle z, b z\rangle_{C} \neq 0$. Surjectivity follows from fact that $p C p$ is spanned by $p\langle m, n\rangle_{C} p$ and equality $p\langle m, n\rangle_{C} p=\Phi\left({ }_{B}\langle m, z\rangle_{B}\langle n, z\rangle\right)$. Also $\Phi\left(b_{1} b_{2}\right)=\Phi\left(b_{1}\right) \Phi\left(b_{2}\right)$.

To prove statement it remains to check that $\langle u, v\rangle_{A}=\Psi(u)^{*} \Psi(v), \Phi\left({ }_{B}\langle u, v\rangle\right)=$ $\Psi(u) \Psi(v)^{*}$ and $\Phi\left({ }_{B}\langle u, v\rangle\right) \Psi(t)=\Psi\left({ }_{B}\langle u, v\rangle t\right)$. Indeed,

$$
\Psi(u)^{*} \Psi(v)=\sum_{i}\left\langle u, z_{i}\right\rangle_{A}\left\langle z_{i}, v\right\rangle_{A}=\left\langle u,{ }_{B}\langle z, z\rangle v\right\rangle_{A}=\langle u, v\rangle_{A} .
$$

Next, we compare $(i, j)^{\prime}$ th matrix entry for $\Phi\left({ }_{B}\langle u, v\rangle\right)=\left\langle z,{ }_{B}\langle u, v\rangle z\right\rangle_{C}$ and $\Psi(u) \Psi(v)^{*}$ :

$$
\left\langle z_{i},{ }_{B}\langle u, v\rangle z_{j}\right\rangle_{A}=\left\langle z_{i}, u\right\rangle_{A}\left\langle v, z_{j}\right\rangle_{A} .
$$

Now we compare $i$ 'th coordinate in $\Phi\left({ }_{B}\langle u, v\rangle\right) \Psi(t)$ and $\Psi\left({ }_{B}\langle u, v\rangle t\right)$ :

$$
\left(\Psi(u) \Psi(v)^{*} \Psi(t)\right)_{i}=\Psi(u)_{i}\langle v, t\rangle_{A}=\left\langle z_{i}, u\right\rangle_{A}\langle v, t\rangle_{A}=\left\langle z_{i},{ }_{B}\langle u, v\rangle t\right\rangle_{A} .
$$

Corollary 3.1. Suppose there are two structures of a $B-A$-imprimitivity bimodule on bimodule $M:_{B}\langle\cdot, \cdot\rangle^{i}$ and $\langle\cdot, \cdot\rangle_{A}^{i}$ for $i=1,2$. If $\langle\cdot, \cdot\rangle_{A}^{1}=\langle\cdot, \cdot\rangle_{A}^{2}$ then also ${ }_{B}\langle\cdot, \cdot\rangle^{1}={ }_{B}\langle\cdot, \cdot\rangle^{2}$, and vice versa.

Proof. Due to theorem above it is sufficient to check the statement in case $M=$ $p A^{\infty}$ and $\langle x, y\rangle_{A}^{1}=\langle x, y\rangle_{A}^{2}=x^{*} y$. Then for any $z \in p A^{\infty}$ we have ${ }_{B}\langle x, y\rangle z=$ $x\langle y, z\rangle_{A}=x y^{*} z$. Taking $z=p_{k}$ for all columns of $p=\left(p_{k}\right)$ we get ${ }_{B}\langle x, y\rangle=$ ${ }_{B}\langle x, y\rangle p=x y^{*} p=x y^{*}$, so the second inner product is defined by the first one.

\section{Composition of strong Morita morphisms}

Evidently choice of inner products for an $A-B$-imprimitivity bimodule $M$ is nonunique. For example, we can multiply them both by positive number and with such new inner products $M$ will be again an $A-B$-imprimitivity bimodule. Anyway, following theorem gives one natural choice of imprimitivity bimodule structure on tensor product of two imprimitivity bimodules.

Theorem 2. Let $A, B, C$ be unital $C^{*}$-algebras, $M, N$ be $A-B$ and $B-C$ imprimitivity bimodules correspondingly. Then $M \underset{B}{\otimes} N$ with inner products defined by

$$
\begin{gathered}
\langle x \otimes z, y \otimes t\rangle_{C}=\left\langle z,\langle x, y\rangle_{B} t\right\rangle_{C} \\
{ }_{A}\langle x \otimes z, y \otimes t\rangle={ }_{A}\left\langle x_{B}\langle z, t\rangle, y\right\rangle
\end{gathered}
$$

is an $A-C$-imprimitivity bimodule.

Proof. Let $K=M \underset{B}{\otimes} N$. We check that $K$ is a right $C$-rigged space. First, let us see that $\langle\cdot, \cdot\rangle_{C}$ on $K$ is well-defined $C$-valued inner product antilinear in first variable. Indeed, for $b \in B$

$$
\begin{gathered}
\langle x b \otimes z, y \otimes t\rangle_{C}=\left\langle z,\langle x b, y\rangle_{B} t\right\rangle_{C}=\left\langle z, b^{*}\langle x, y\rangle_{B} t\right\rangle_{C} \\
=\left\langle b z,\langle x, y\rangle_{B} t\right\rangle_{C}=\langle x \otimes b z, y \otimes t\rangle_{C} .
\end{gathered}
$$

Taking $b \in \mathbb{C} 1$ we see that $\langle\cdot, \cdot\rangle_{C}$ is antilinear in first variable. Analogously $\langle x \otimes z, y b \otimes t\rangle_{C}=\langle x \otimes z, y \otimes b t\rangle_{C}$ and $\langle\cdot, \cdot\rangle_{C}$ is linear in second variable. To see positivity of $\left\langle\sum_{i=1}^{n} x_{i} \otimes z_{i}, \sum_{i} x_{i} \otimes z_{i}\right\rangle_{C}=\sum_{i, j}\left\langle z_{i},\left\langle x_{i}, x_{j}\right\rangle_{B} z_{j}\right\rangle_{C}$, we recall that $M^{n}$ is an $A-\mathcal{M}_{n} B$-imprimitivity bimodule. So matrix $H=\left\langle x_{i}, x_{j}\right\rangle_{B}$ is positive 
element of $\mathcal{M}_{n} B$. Thus $\langle y, H y\rangle_{C}=\sum_{i, j}\left\langle y_{i}, h_{i, j} y_{j}\right\rangle_{C} \geq 0$ as $N^{n}$ is an $\mathcal{M}_{n} B-C$ imprimitivity bimodule.

Consider elements $x_{i}, y_{i}$ in $M$ such that $\sum_{i}\left\langle x_{i}, y_{i}\right\rangle_{B}=1$. Then for any $z, t \in N$

$$
\sum_{i}\left\langle x_{i} \otimes z, y_{i} \otimes t\right\rangle_{C}=\langle z, t\rangle_{C},
$$

so $\operatorname{Im}\langle\cdot, \cdot\rangle_{C}$ on $N$ is a subset of $\operatorname{Im}\langle\cdot, \cdot\rangle_{C}$ on $K$. Thus $\operatorname{Im}\langle\cdot, \cdot\rangle_{C}$ on $K$ is dense in $C$.

For $c \in C$ we obviously have relation

$$
\langle x \otimes z, y \otimes t c\rangle_{C}=\left\langle z,\langle x, y\rangle_{B} t c\right\rangle_{C}=\langle x \otimes z, y \otimes t\rangle_{C} c,
$$

so we proved $K$ is a right $C$-rigged space. Analogously $K$ is a left $A$-rigged space.

Now we check condition (2) in Definition 1.3 :

$$
\begin{aligned}
v \otimes w\langle x \otimes z, y \otimes t\rangle_{C} & =v \otimes w\left\langle\langle y, x\rangle_{B} z, t\right\rangle_{C}=v \otimes{ }_{B}\left\langle w,\langle y, x\rangle_{B} z\right\rangle t \\
=v_{B}\left\langle w,\langle y, x\rangle_{B} z\right\rangle \otimes t & =v_{B}\langle w, z\rangle\langle x, y\rangle_{B} \otimes t={ }_{A}\left\langle v_{B}\langle w, z\rangle, x\right\rangle y \otimes t \\
& ={ }_{A}\langle v \otimes w, x \otimes z\rangle y \otimes t .
\end{aligned}
$$

For condition (3) consider $a \in A$ and

$$
\begin{gathered}
\left\langle a \sum_{i=1}^{n} x_{i} \otimes z_{i}, a \sum_{i} x_{i} \otimes z_{i}\right\rangle_{C}=\sum_{i, j}\left\langle z_{i},\left\langle a x_{i}, a x_{j}\right\rangle_{B} z_{j}\right\rangle_{C} \\
\leq \sum_{i, j}\left\langle z_{i},\|a\|^{2}\left\langle x_{i}, x_{j}\right\rangle_{B} z_{j}\right\rangle_{C}=\|a\|^{2}\left\langle\sum_{i} x_{i} \otimes z_{i}, \sum_{i} x_{i} \otimes z_{i}\right\rangle_{C}
\end{gathered}
$$

as $M^{n}$ is an $A-\mathcal{M}_{n} B$-imprimitivity bimodule. Analogously we can check condition (3) for ${ }_{A}\langle\cdot, \cdot\rangle$.

We remark that this statement is also true in case of unital pre- $C^{*}$-algebras. The proof is the same just we need additional continuity arguments to prove density of images of pre-inner products.

\section{Morita Bimodules OVER QUANTUM TORI}

Recall that quantum torus $A_{\theta}$ for $\theta \in \mathbb{R} \backslash \mathbb{Q}$ is a transformation group $C^{*}$-algebra $C^{*}(\theta \mathbb{Z}, \mathbb{R} / \mathbb{Z})$. It is known that $A_{\theta}$ is universal $C^{*}$-algebra generated by two unitaries $U, V \in A_{\theta}$ satisfying relation $U V=e(\theta) V U$. The choice of such unitaries is not unique. If $U, V \in A_{\theta}$ are chosen we call them a frame.

From Example 1.6 we see that $A_{\theta}=C^{*}(\theta \mathbb{Z}, \mathbb{R} / \mathbb{Z})$ is strongly Morita equivalent to $C^{*}(\mathbb{Z}, \mathbb{R} / \theta \mathbb{Z}) \cong C^{*}\left(\frac{1}{\theta} \mathbb{Z}, \mathbb{R} / \mathbb{Z}\right)=A_{\frac{1}{\theta}}$. Obviously $A_{\theta+1}=A_{\theta}$, as relation $U V=$ $e(\theta) V U$ is invariant under transformation $\theta \mapsto \theta+1$. Also $A_{\theta} \cong A_{-\theta}$ as we can map $U$ to $V^{\prime}$ and $V$ to $U^{\prime}$ for any frames $U, V \in A_{\theta}, U^{\prime}, V^{\prime} \in A_{\theta}$. Recall that $G L_{2}(\mathbb{Z})$ acts on complex numbers by

$$
\left(\begin{array}{ll}
a & b \\
c & d
\end{array}\right) \theta=\frac{a \theta+b}{c \theta+d}
$$

So we see that $A_{\theta}$ is strongly Morita equivalent to $A_{g \theta}$ for any $g \in G L_{2}(\mathbb{Z})$. Indeed, as $G L_{2}(\mathbb{Z})$ is generated by $\left(\begin{array}{ll}0 & 1 \\ 1 & 0\end{array}\right)$ and $\left(\begin{array}{ll}1 & 1 \\ 0 & 1\end{array}\right)$, its orbit is generated by transformations $\theta \mapsto \theta+1$ and $\theta \mapsto \frac{1}{\theta}$. Conversely, it is shown in [11] that $A_{\theta}$ and $A_{\theta^{\prime}}$ are not strongly Morita equivalent if $\theta$ and $\theta^{\prime}$ don't lie in the same orbit of $G L_{2}(\mathbb{Z})$.

Below we recall an explicit construction of $A_{g \theta}-A_{\theta}$-imprimitivity bimodule $E(g, \theta)$ for $g \in S L_{2}(\mathbb{Z}), \theta \in \mathbb{R} \backslash \mathbb{Q}([2],[3],[1])$. (Bimodules for $g \in G L_{2}(\mathbb{Z})$ can be easily obtained from those by composition with homomorphism $U \mapsto V^{\prime}, V \mapsto U^{\prime}$ of quantum tori on the left.) It is proven in [2] that $E(h g, \theta) \cong E(h, g \theta) \underset{A_{g \theta}}{\otimes} E(g, \theta)$ 
as bimodule, and we claim in theorem below that inner products satisfy relations of Theorem 2

To construct our bimodules we need to fix a frame in $A_{\theta}$ for each $\theta \in \mathbb{R} \backslash \mathbb{Q}$. (If $\theta=\theta^{\prime}$ modulo $\mathbb{Z}$ then the frames should coincide.) Let $g=\left(\begin{array}{ll}a & b \\ c & d\end{array}\right) \in S L_{2}(\mathbb{Z})$. If $c=0$ we put $E(g, \theta)=A_{\theta}$ with action of $A_{g \theta}=A_{\theta}$ via multiplication from the left and action of $A_{\theta}$ by right multiplication, and define inner products by $A_{g \theta}\langle a, b\rangle=a b^{*}$ and $\langle a, b\rangle_{A_{\theta}}=a^{*} b$ as in Example 1.5. If $c \neq 0$, we consider the space $E^{0}(g, \theta)=S(\mathbb{R} \times \mathbb{Z} / c \mathbb{Z})$ with following actions of generators $U, V$ of $A_{\theta}$ and $U^{\prime}, V^{\prime}$ of $A_{g \theta}$ on $f \in E^{0}(g, \theta)$ :

$$
\begin{gathered}
(f U)(x, \alpha)=f\left(x-\frac{c \theta+d}{c}, \alpha-1\right) \\
(f V)(x, \alpha)=e\left(x-\alpha \frac{d}{c}\right) f(x, \alpha) \\
\left(U^{\prime} f\right)(x, \alpha)=f\left(x-\frac{1}{c}, \alpha-a\right) \\
\left(V^{\prime} f\right)(x, \alpha)=e\left(\frac{x}{c \theta+d}-\frac{\alpha}{c}\right) f(x, \alpha)
\end{gathered}
$$

We define for $f, s \in E^{0}(g, \theta)$ inner products:

$$
\begin{gathered}
A_{g \theta}\langle f, s\rangle=\sum_{n \in \mathbb{Z}^{2}}\left\langle f, U^{\prime} n_{1} V^{\prime} n_{2} s\right\rangle_{L_{2}} U^{\prime} n_{1} V^{\prime} n_{2} \\
\langle f, s\rangle_{A_{\theta}}=\frac{1}{c \theta+d} \sum_{n \in \mathbb{Z}^{2}}\left\langle s, f U^{n_{1}} V^{n_{2}}\right\rangle_{L_{2}} U^{n_{1}} V^{n_{2}}
\end{gathered}
$$

Let $E(g, \theta)$ be the completion of $E^{0}(g, \theta)$ with the norm $\|f\|=\left\|A_{g \theta}\langle f, f\rangle\right\|^{\frac{1}{2}}$. Then $E(g, \theta)$ is an $A_{g \theta}-A_{\theta}$-imprimitivity bimodule (Theorem 3.2 in [1]).

In [2, [3] there are constructed bimodule isomorphisms $t_{h, g}: E(h, g \theta) \underset{A_{g \theta}}{\otimes} E(g, \theta) \rightarrow$ $E(h g, \theta)$ for $h, g \in S L_{2}(\mathbb{Z})$.

Theorem 3. For $h, g \in S L_{2}(\mathbb{Z}), f_{1}, s_{1} \in E(h, g \theta)$ and $f_{2}, s_{2} \in E(g, \theta)$

$$
\begin{aligned}
A_{h g \theta}\left\langle t_{h, g}\left(f_{1} \otimes f_{2}\right), t_{h, g}\left(s_{1} \otimes s_{2}\right)\right\rangle & =A_{h g \theta}\left\langle f_{1} A_{g \theta}\left\langle f_{2}, s_{2}\right\rangle, s_{1}\right\rangle \\
\left\langle t_{h, g}\left(f_{1} \otimes f_{2}\right), t_{h, g}\left(s_{1} \otimes s_{2}\right)\right\rangle_{A_{\theta}} & =\left\langle f_{2},\left\langle f_{1}, s_{1}\right\rangle_{A_{g \theta}} s_{2}\right\rangle_{A_{\theta}}
\end{aligned}
$$

Proof. First, due to Theorem 2 and Corollary 3.1 it is enough to check only one of two statements of the theorem. We prefer the second one.

As maps $t_{h, g}$ are associative (Proposition 1.2 in [3]) it is enough to check the statement for generators of $S L_{2}(\mathbb{Z})$ at place of $h$ only. Indeed, suppose the statement is true for $E\left(h_{1}, g \theta\right) \otimes E(g, \theta), E\left(h_{2}, h_{1} g \theta\right) \otimes E\left(h_{1} g, \theta\right)$ and $E\left(h_{2}, h_{1} g \theta\right) \otimes$ $E\left(h_{1}, g \theta\right)$. Then it is true for $E\left(h_{2} h_{1}, g \theta\right) \otimes E(g, \theta)$ due to associativity relation

$$
t_{h_{2} h_{1}, g} \circ\left(t_{h_{2}, h_{1}} \otimes i d\right)=t_{h_{2}, h_{1} g} \circ\left(i d \otimes t_{h_{1}, g}\right) .
$$

Take $h=\left(\begin{array}{ll}1 & 1 \\ 0 & 1\end{array}\right)$. Then $f_{1}, s_{1} \in A_{g \theta},\left\langle f_{1}, s_{1}\right\rangle_{A_{g \theta}}=f_{1}^{*} s_{1}, t_{h, g}\left(f_{1}, f_{2}\right)=f_{1} f_{2}($ in sense of left action) and similar $t_{h, g}\left(s_{1}, s_{2}\right)=s_{1} s_{2}$. As $h\left(\begin{array}{ll}a & b \\ c & d\end{array}\right)=\left(\begin{array}{cc}a+c & b+d \\ c & d\end{array}\right)$ we have no changes in formulas for action of quantum tori, so $E(h g, \theta)=E(g, \theta)$ and

$$
\left\langle f_{1} f_{2}, s_{1} s_{2}\right\rangle_{A_{\theta}}=\left\langle f_{2}, f_{1}^{*} s_{1} s_{2}\right\rangle_{A_{\theta}}
$$

as $E(g, \theta)$ is an $A_{g \theta}-A_{\theta}$-imprimitivity bimodule. Indeed, for an $A-B$-imprimitivity bimodule $M$ we have $\langle a x, y\rangle_{B}=\left\langle x, a^{*} y\right\rangle_{B}$ for $a \in A, x, y \in M$. 
Now take $h=\left(\begin{array}{cc}0 & -1 \\ 1 & 0\end{array}\right)$. Then $h g=\left(\begin{array}{cc}-c & -d \\ a & b\end{array}\right)$. Let us consider the case $g \neq h, c \neq 0$. Cases $g=h$ and $c=0$ can be done analogously. Obviously we can restrict to dense set of Schwartz functions $f_{1}, s_{1} \in E^{0}(h, g \theta), f_{2}, s_{2} \in E^{0}(g, \theta)$. $E^{0}(h, g \theta)=S(\mathbb{R})$ with

$$
\left\langle f_{1}, s_{1}\right\rangle_{A_{g \theta}}=\frac{1}{g \theta} \sum_{n \in \mathbb{Z}^{2}} \int s_{1}(y) e\left(-y n_{2}\right) \bar{f}_{1}\left(y-n_{1} \theta\right) d y U^{n_{1}} V^{n_{2}}
$$

where $U, V \in A_{g \theta}$. Let $U^{\prime}, V^{\prime}$ be generators of $A_{\theta}$. Comparing coefficients near $U^{\prime} m_{1} V^{\prime} m_{2}$ in identity, which we need to prove, we see that it is equivalent to

$\frac{1}{a \theta+b}\left\langle t_{h, g}\left(s_{1} \otimes s_{2}\right), t_{h, g}\left(f_{1} \otimes f_{2} U^{\prime} m_{1} V^{\prime} m_{2}\right)\right\rangle_{L_{2}}=\frac{1}{c \theta+d}\left\langle\left\langle f_{1}, s_{1}\right\rangle_{A_{g \theta}} s_{2}, f_{2} U^{\prime} m_{1} V^{\prime} m_{2}\right\rangle_{L_{2}}$

Substituting $f_{2}$ instead of $f_{2} U^{\prime} m_{1} V^{\prime} m_{2}$, we need to prove for arbitrary $f_{1}, s_{1} \in S(\mathbb{R})$, $f_{2}, s_{2} \in S(\mathbb{R} \times \mathbb{Z} / c \mathbb{Z})$

$\left\langle t_{h, g}\left(s_{1} \otimes s_{2}\right), t_{h, g}\left(f_{1} \otimes f_{2}\right)\right\rangle_{L_{2}}=\sum_{n \in \mathbb{Z}^{2}} \int s_{1}(y) e\left(-y n_{2}\right) \bar{f}_{1}\left(y-n_{1} \theta\right) d y\left\langle U^{n_{1}} V^{n_{2}} s_{2}, f_{2}\right\rangle_{L_{2}}$

This is a routine computation using Poisson summation formula. We use abbreviations LHS (RHS) for left-(right)-hand side of this identity correspondingly. By explicit formula for $t_{h, g}$ (Proposition 1.2 in [3])

$$
t_{h, g}\left(s_{1} \otimes s_{2}\right)(x, \alpha)=\sum_{n \in \mathbb{Z}} s_{1}\left(\frac{x}{c \theta+d}+g \theta\left(\frac{c b}{a} \alpha-n\right)\right) s_{2}\left(x-\frac{b}{a} \alpha+\frac{n}{c}, a n\right),
$$

and analogously for $t_{h, g}\left(f_{1} \otimes f_{2}\right)$. Now

$$
L H S=\sum_{n, m \in \mathbb{Z}} \sum_{\alpha \in \mathbb{Z} / a \mathbb{Z}} \int s_{1}(z) s_{2}\left(y-\frac{m-n}{c}, a n\right) \bar{f}_{1}(z-g \theta(m-n)) \bar{f}_{2}(y, a m) d y
$$

where $z=\frac{x}{c \theta+d}+g \theta\left(\frac{c b}{a} \alpha-n\right)$ and $y=x-\frac{b}{a} \alpha+\frac{m}{c}$. Let us represent $m=d m_{1}+c m_{2}$ with $m_{1} \in \mathbb{Z} / c \mathbb{Z}$ and $m_{2} \in \mathbb{Z}$. Then $a m=m_{1}$ and $a n=m_{1}-a(m-n)$ modulo $c$. Introducing new variable $n_{1}=m-n$ we proceed:

$$
=\sum_{m_{1} \in \mathbb{Z} / c \mathbb{Z}} \int \sum_{n_{1} \in \mathbb{Z}} \sum_{m_{2} \in \mathbb{Z}, \alpha \in \mathbb{Z} / a \mathbb{Z}} s_{1}(z) \bar{f}_{1}\left(z-g \theta n_{1}\right)\left(U^{n_{1}} s_{2}\right)\left(y, m_{1}\right) \bar{f}_{2}\left(y, m_{1}\right) d y
$$

Let us express $z$ via $y$ and summing variables:

$$
\begin{gathered}
z=\frac{1}{c \theta+d}\left(y+\frac{b}{a} \alpha-\frac{m}{c}\right)+g \theta\left(\frac{c b}{a} \alpha-n\right) \\
=\frac{1}{c \theta+d}\left(y+\frac{b}{a} \alpha-m_{2}-\frac{d}{c} m_{1}\right)+\frac{a \theta+b}{c \theta+d}\left(\frac{c b}{a} \alpha-c m_{2}-d m_{1}+n_{1}\right) \\
=\left(b \alpha-m_{2} a\right)-\frac{a d}{c} m_{1}+\frac{1}{c \theta+d}\left(y+(a \theta+b) n_{1}\right)
\end{gathered}
$$

Denote $n_{2}=b \alpha-m_{2} a$, and $z_{0}=z-n_{2}$. Then by Poisson summation formula

$$
\sum_{n_{2} \in \mathbb{Z}} s_{1}\left(n_{2}+z_{0}\right) \bar{f}_{1}\left(n_{2}+z_{0}-g \theta n_{1}\right)=\sum_{n_{2} \in \mathbb{Z}} e\left(z_{0}\right)^{n_{2}} \int e\left(-t n_{2}\right) s_{1}(t) \bar{f}_{1}\left(t-g \theta n_{1}\right) d t .
$$

We put this into LHS, and note that $e\left(z_{0}\right)^{n_{2}}\left(U^{n_{1}} s_{2}\right)\left(y, m_{1}\right)=\left(U^{n_{1}} V^{n_{2}} s_{2}\right)\left(y, m_{1}\right)$. So LHS =

$$
\sum_{m_{1} \in \mathbb{Z} / c \mathbb{Z}} \int \sum_{n_{1}, n_{2} \in \mathbb{Z}} \int e\left(-t n_{2}\right) s_{1}(t) \bar{f}_{1}\left(t-g \theta n_{1}\right) d t\left(U^{n_{1}} V^{n_{2}} s_{2}\right)\left(y, m_{1}\right) \bar{f}_{2}\left(y, m_{1}\right) d y
$$




$$
=\sum_{n_{1}, n_{2}} \int e\left(-t n_{2}\right) s_{1}(t) \bar{f}_{1}\left(t-g \theta n_{1}\right) d t\left\langle U^{n_{1}} V^{n_{2}} s_{2}, f_{2}\right\rangle_{L_{2}}=R H S
$$

\section{Real multiplication}

Irrational number $\theta \in \mathbb{R} \backslash \mathbb{Q}$ is a root of quadratic equation if and only if there exist matrix $g \in S L_{2}(\mathbb{Z}), g \neq \pm 1$ such that $g \theta=\theta$. Let us fix such $g$ and $\theta$. It follows from Section 5 that there are nontrivial $A_{\theta}-A_{\theta}$-imprimitivity bimodules exactly in this case. Now we are going to construct a graded ring $R=R(g, \theta)=$ $\oplus R_{n}$ using tensor products and inner products in these imprimitivity bimodules. $n \geq 1$

We start with construction of another graded ring due to Polishchuk 4, which uses only tensor products.

We consider the set of bimodules $E\left(g^{n}, \theta\right), n \geq 1$ defined in previous section, and have the family of isomorphisms

$$
t_{g^{m}, g^{n}}: E\left(g^{n}, \theta\right) \otimes E\left(g^{m}, \theta\right) \widetilde{\rightarrow} E\left(g^{n+m}, \theta\right) .
$$

Let $\mathbb{H}_{k}=\left\{M \in \mathcal{M}_{k} \mathbb{C} \mid M=M^{t}\right.$ and $\left.\Im(M)>0\right\}$ be so-called Siegel upper halfplane. So, $\mathbb{H}_{1}$ is just an upper half of complex plane $\mathbb{C}$, and we fix $\tau \in \mathbb{H}_{1}$. Denote matrix entries of $g^{n}$ by $\left(\begin{array}{ll}a_{n} & b_{n} \\ c_{n} & d_{n}\end{array}\right)$. Denote $\mu_{n}=\tau \frac{c_{n}}{c_{n} \theta+d_{n}}$. Note that $c_{n} \theta+d_{n}$ is an eigenvalue of $g^{n}$, so it is nonzero. Also $c_{n} \neq 0$ as $g^{n}$ is a nontrivial matrix stabilizing $\theta$. Thus $\mu_{n} \neq 0$. Denote

$$
E_{n}=\left\{\begin{array}{ll}
\left\{\phi_{f}(x, \alpha)=e\left(\mu_{n} \frac{x^{2}}{2}\right) f(\alpha) \mid f: \mathbb{Z} / c_{n} \mathbb{Z} \rightarrow \mathbb{C}\right\}, & \frac{c_{n}}{c_{n} \theta+d_{n}}>0 \\
\{0\}, & \frac{c_{n}}{c_{n} \theta+d_{n}}<0
\end{array} \subset E\left(g^{n}, \theta\right) .\right.
$$

$E_{n}$ is either 0 or a $\left|c_{n}\right|$-dimensional vector space. In fact we have either $E_{n}=\{0\}$ for all $n$ or $E_{n} \neq\{0\}$ for all $n$. Indeed, we see that definition of $E_{n}$ is the same for $E(g, \theta)$ and $E(-g, \theta)$. Thus taking either $g$ or $-g$ instead of $g$ we can suppose that $c_{1} \theta+d_{1}>0$. $c_{1} \theta+d_{1}$ is an eigenvalue of $g$, so $g$ has positive eigenvalues. Now it follows from $\sum_{n=1}^{\infty} c_{n} t^{n}=\frac{c t}{t^{2}-\operatorname{tr}(g) t+1}$ that all $c_{n}$ have the same sign, as all coefficients of power series for $\frac{1}{t^{2}-\operatorname{tr}(g) t+1}$ are positive. All $c_{n} \theta+d_{n}$ are eigenvalues of $g^{n}$, so they are also positive.

Consider the set

$$
S_{\theta}:=\left\{g=\left(\begin{array}{ll}
a & b \\
c & d
\end{array}\right) \in S L_{2}(\mathbb{Z}) \mid g \neq \pm 1, g \theta=\theta, \operatorname{tr}(g)>0 \& c>0\right\}
$$

It is always nonempty: we already showed how to satisfy first three conditions, then if the forth is not satisfied we can take $g^{-1}$ instead of $g$.

Further we suppose $g \in S_{\theta}$. Then all $E_{n}$ are nonzero vector spaces. It was noticed already in [2] that vector spaces $E_{n}$ are preserved under tensor products of bimodules. Following can be checked by direct computation:

Proposition 6.1. For $f: \mathbb{Z} / c_{n} \mathbb{Z} \rightarrow \mathbb{C}, g: \mathbb{Z} / c_{m} \mathbb{Z} \rightarrow \mathbb{C}$ we have $t_{g^{n}, g^{m}}\left(\phi_{f} \otimes \phi_{g}\right)=$ $\phi_{f \underset{n, m}{\star} g}$ where

$$
f \underset{n, m}{\star \star} g(\alpha)=\sum_{q \in \mathbb{Z}} e\left(\frac{\tau}{2} \frac{c_{n+m}}{c_{n} c_{m}}\left(q-\frac{c_{m} d_{n+m}}{c_{n+m}} \alpha\right)^{2}\right) f\left(a_{n} d_{n+m} \alpha-q\right) g\left(a_{m} q\right)
$$

is a function on $\mathbb{Z} / c_{n+m} \mathbb{Z}$. 
Now we consider the graded ring $E=\oplus_{n \geq 1} E_{n}$ with multiplication given by $\phi_{f} * \phi_{g}:=\phi_{t \underset{n, m}{\star} g} \in E_{n+m}$ for $\phi_{f} \in E_{n}, \phi_{g} \in E_{m}$. Associativity of this multiplication follows from identity

$$
t_{g^{n+m}, g^{k}} \circ\left(t_{g^{n}, g^{m}} \otimes i d\right)=t_{g^{n}, g^{m+k}} \circ\left(i d \otimes t_{g^{m}, g^{k}}\right): E_{n} \otimes E_{m} \otimes E_{k} \rightarrow E_{n+m+k}
$$

stated in Proposition 1.2 in [3]. Note that if we choose for basis in $E_{n}$ functions of the form $\phi_{f}$ with characters $f \in\left(\mathbb{Z} / c_{n} \mathbb{Z}\right)^{*}$, we would get multiplication table consisting of values at rational points of various theta functions with rational characters $\theta\left[\begin{array}{l}\alpha \\ \beta\end{array}\right](\gamma, \delta \tau)$ where $\alpha, \beta, \gamma, \delta \in \mathbb{Q}$ (see, e.g. [12]). For example,

$$
1 \underset{n, m}{\star} 1(\alpha)=\theta\left[\begin{array}{r}
\frac{c_{m} d_{n+m}}{c_{n+m}} \alpha \\
0
\end{array}\right]\left(0, \frac{\tau}{2} \frac{c_{n+m}}{c_{n} c_{m}}\right) .
$$

In [4] (Theorem 2.4) there are established criterions whether the ring $E$ is generated over $\mathbb{C}$ by $E_{1}$, is quadratic and is Koszul. Using them we state the criterion whether there exist $g \in S_{\theta}$ such that $E$ have these good properties:

Theorem 4. Let $\theta \in \mathbb{R} \backslash \mathbb{Q}$ be a quadratic irrationality, and $\theta^{\prime}$ be its Galois conjugate. Then the following conditions are equivalent:

(1) $\left|\theta-\theta^{\prime}\right|<1$;

(2) there exist $g \in S_{\theta}$ such that the ring $E$ is generated by $E_{1}$ over $\mathbb{C}$;

(3) there exist $g \in S_{\theta}$ such that the ring $E$ is quadratic;

(4) there exist $g \in S_{\theta}$ such that the ring $E$ is Koszul.

Proof. First we show (2),(3) and (4) imply (1). Let $g=\left(\begin{array}{ll}a & b \\ c & d\end{array}\right)$ with given properties exist. As $g \in S_{\theta}$ then it satisfies conditions of Theorem 2.4 in 4 . This implies $c \geq a+d+\varepsilon$, where $\varepsilon=0$ for (2), $\varepsilon=1$ for (3), $\varepsilon=2$ for (4). Then, as $c \theta^{2}+(d-a) \theta-b=0$,

$$
\left|\theta-\theta^{\prime}\right|^{2}=\frac{(d-a)^{2}+4 b c}{c^{2}}=\frac{(d+a)^{2}-4}{c^{2}} \leq \frac{(d+a)^{2}-4}{(d+a)^{2}}<1 .
$$

Let us prove that (1) implies (2),(3) and (4). Namely, we are going to show that (i) implies that for every $\varepsilon \leq 2$ there exist $g \in S_{\theta}$ such that $c>a+d+\varepsilon$. This will imply (2) for $\varepsilon=1,(3)$ and (4) for $\varepsilon=2$ due to Theorem 2.4 in 4 .

Take any $g \in S_{\theta}$. Now, as $g$ stabilizes $\theta$, we have norm and trace

$$
N(\theta)=-\frac{b}{c}=\frac{1-a d}{c^{2}}, \operatorname{Tr}(\theta)=\frac{a-d}{c},
$$

and

$$
(a+d)^{2}=(a-d)^{2}+4 a d=c^{2}\left(\operatorname{Tr}(\theta)^{2}-4 N(\theta)\right)+4=c^{2}\left|\theta-\theta^{\prime}\right|^{2}+4 .
$$

So, as $\left|\theta-\theta^{\prime}\right|<1$ we have $(a+d)^{2}<(c-\varepsilon)^{2}$ if $c$ is large enough, and $a+d<c-\varepsilon$, because $a+d>2$ and $\varepsilon \leq 2$ and $c>0$. Then one can take $g^{n}$, which also belongs to $S_{\theta}$, instead of $g$, and get large enough number $c$ in the last identity.

Now we are going to construct another ring, which also uses inner products in imprimitivity bimodules $E\left(g^{n}, \theta\right)$. We will use left $A_{\theta}$-valued inner products, but the same construction can be done for the right ones. We put $R_{n}=\left.\operatorname{Im}_{A_{\theta}}\langle\cdot, \cdot\rangle\right|_{E_{n}}-$ the vector space of finite sums of values of left inner product on pairs of vectors from 
$E_{n} \subset E\left(g^{n}, \theta\right)$. In Introduction we defined for $\Omega \in \mathbb{H}_{2}$ and function $f: \mathbb{Z}^{2} \rightarrow \mathbb{C}$ periodic w.r.t. some cofinite lattice in $\mathbb{Z}^{2}$ an element

$$
\Theta[f](\Omega)=\sum_{\vec{m} \in \mathbb{Z}^{2}} f(\vec{m}) e\left(\frac{1}{2} \vec{m}^{t} \Omega \vec{m}\right) e\left(-\frac{\theta}{2} m_{1} m_{2}\right) U^{m_{1}} V^{m_{2}} \in A_{\theta}
$$

Proposition 6.2. $R_{n}=\left\{\Theta[f]\left(\frac{1}{c_{n}\left(c_{n} \theta+d_{n}\right)} \Omega\right) \mid f: \mathbb{Z}^{2} / c_{n} \mathbb{Z}^{2} \rightarrow \mathbb{C}\right\}$ where

$$
\Omega=\frac{i}{2 \Im \tau}\left(\begin{array}{cc}
|\tau|^{2} & -\Re \tau \\
-\Re \tau & 1
\end{array}\right) \in \mathbb{H}_{2}
$$

Proof. By routine computation we get

$$
A_{\theta}\left\langle\phi_{f}, \phi_{g}\right\rangle=\frac{1}{2\left(\Im \mu_{n}\right)} \sum_{\vec{m} \in \mathbb{Z}^{2}} Q(\vec{m}) e\left(\frac{1}{2} \vec{m}^{t} \frac{\Omega}{c_{n}\left(c_{n} \theta+d_{n}\right)} \vec{m}\right) e\left(-\frac{\theta}{2} m_{1} m_{2}\right) U^{m_{1}} V^{m_{2}}
$$

where

$$
Q(\vec{m})=\sum_{\alpha \in \mathbb{Z} / c_{n} \mathbb{Z}} f\left(\alpha+a_{n} m_{1}\right) \bar{g}(\alpha) e\left(\frac{\alpha}{c_{n}} m_{2}\right) .
$$

Now the statement follows. We have $\frac{1}{c_{n}\left(c_{n} \theta+d_{n}\right)} \Omega \in \mathbb{H}_{2}$ since $\Omega \in \mathbb{H}_{2}$ and $c_{n}\left(c_{n} \theta+\right.$ $\left.d_{n}\right)>0$ as $g \in S_{\theta}$.

Note, that $R_{n}$ is a vector space. $\operatorname{dim} R_{n}=c_{n}^{2}=\left(\operatorname{dim} E_{n}\right)^{2}$, what implies in particular that there are no linear relations among $A_{\theta}\left\langle\phi_{f_{i}}, \phi_{f_{j}}\right\rangle$ for any basis $\left\{f_{i}\right\}$ in space of functions on $\mathbb{Z} / c_{n} \mathbb{Z}$.

Now we define an operation $\underset{n, m}{\star}: R_{n} \otimes R_{m} \rightarrow R_{n+m}$ :

$$
\sum_{i} A_{\theta}\left\langle x_{i}, y_{i}\right\rangle \underset{n, m}{\star} \sum_{j} A_{\theta}\left\langle z_{j}, t_{j}\right\rangle:=\sum_{i, j} A_{\theta}\left\langle x_{i} * z_{j}, y_{i} * t_{j}\right\rangle
$$

This operation is well defined. Indeed, every element of $R_{n}$ can be uniquely represented as a linear combination of $A_{\theta}\left\langle\phi_{f_{i}}, \phi_{f_{j}}\right\rangle$ as we remarked above. We can now introduce the ring $R=\oplus_{n \geq 1} R_{n}$ with multiplication given by $\phi * \psi:=\underset{n, m}{\star} \psi \epsilon$ $R_{n+m}$ for $\phi \in R_{n}, \psi \in R_{m}$. Multiplication is obviously associative, because it is associative in the ring $E$ defined above. Analogously to Theorem 4 we have:

Theorem 5. Let $\theta \in \mathbb{R} \backslash \mathbb{Q}$ be a quadratic irrationality, $\theta^{\prime}$ be its Galois conjugate and $\left|\theta-\theta^{\prime}\right|<1$. Then there exist such $g \in S_{\theta}$ such that the graded ring $R=R(g, \theta)$ is generated by $R_{1}$ over $\mathbb{C}$.

Proof. By Theorem 4 we can find $g \in S_{\theta}$ such that $E=E(g, \theta)$ is generated by $E_{1}$. So, if we choose some basis $x_{1}, \ldots, x_{c}$ in $E_{1}$, then $E_{n}$ is spanned by the elements $x_{i_{1}} * \cdots * x_{i_{n}}$. Thus $R_{n}$ is spanned by elements

$$
A_{\theta}\left\langle x_{i_{1}} * \cdots * x_{i_{n}}, x_{j_{1}} * \cdots * x_{j_{n}}\right\rangle=\Pi_{s} \quad A_{\theta}\left\langle x_{i_{s}}, x_{j_{s}}\right\rangle
$$

where $A_{\theta}\left\langle x_{i_{s}}, x_{j_{s}}\right\rangle \in R_{1}$.

\section{REFERENCES}

[1] Yu.I.Manin, Real multiplication and noncommutative geometry (ein Alterstraum) // The legacy of Niels Henrik Abel, 685-727, Springer, Berlin, 2004

[2] M.Dieng, A. Schwarz, Differential and complex geometry of two-dimensional noncommutative tori // Lett. Math. Phys. 61 (2002), no. 3, 263-270

[3] A.Polishchuk, A. Schwarz, Categories of holomorphic vector bundles on noncommutative two-tori // Comm. Math. Phys. 236 (2003), no. 1, 135-159

[4] A.Polishchuk, Noncommutative two-tori with real multiplication as noncommutative projective varieties //J. Geom. Phys. 50 (2004), no. 1-4, 162-187

[5] F.P.Boca, Projections in rotation algebras and theta functions // Commun. Math. Phys. 202 (1999), pp. 325-357 
[6] Yu.I.Manin, Quantized theta functions // Common trends in Mathematics and Quantum Field theories (Kyoto, 1990), Progress of Theor. Phys. Supplement, 102 (1990), $219-228$

[7] Yu.I.Manin, Theta functions, quantum tori and Heisenberg groups // EuroConference Moshe Flato 2000, Part III (Dijon), Lett. Math. Phys. 56 (2001), no. 3, 295-320

[8] Yu.I.Manin, Functional equations for quantum theta functions // Publ.Res.Inst.Math.Sci. 40(2004), no.3, $605-624$

[9] M.Rieffel, Induced representations of $C^{*}$-algebras // Advances in Math. 13 (1974), 176 - 257

[10] M.Rieffel, Strong Morita equivalence of certain transformation group $C^{*}$-algebras // Math. Annalen 222 (1976), 7 - 22

[11] M.Rieffel, $C^{*}$-algebras associated with irrational rotations // Pacific Journal of Math. 93 (1981), no. 2, $415-429$

[12] D. Mumford, Tata lectures on theta. I. With the assistance of C. Musili, M. Nori, E. Previato and M. Stillman // Progress in Mathematics, 28. Birkhauser Boston, Inc., Boston, MA, 1983

E-mail address: mariyka@imath.kiev.ua 